\title{
SGLT-2 inhibitors - are they to stay?
}

\author{
Arulmozhi D. Kandasamy*
}

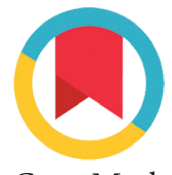

CrossMark

\section{ABSTRACT}

Sodium-glucose co-transporter 2 (SGLT2) inhibitors are a newer class of Type 2 diabetes medications. The drugs are rapidly growing in popularity. In the period between 0ctober 2014 and September 2015, doctors wrote 1.5 million prescriptions for Invokana or Farxiga (dapagliflozin), according to the FDA. They also come in extended formulas and in formulas combined with metformin. These drugs work by preventing the kidneys from reabsorbing sugar and

Keywords: SGLT2 inhibitors, diabetic ketoacidosis, type 2 diabetes

"Corresponding to: Arulmozhi D. Kandasamy, DiscoverSys Inc. 3658 Atkinson Loop SW, Edmonton, Alberta, Canada. Copyright: @ 2016 Arulmozhi D. Kandasamy. This is an open-access article distributed under the terms of the Creative Commons Attribution License.

arul@discoversys.ca

Cite This Article: Kandasamy, A. 2017. SGLT-2 inhibitors - are they to stay?. Diabesity 2(4): 26-27. DOl:10.15562/diabesity.2016.35

\section{INTRODUCTION}

Sodium-glucose co-transporter 2 (SGLT2) inhibitors are a newer class of Type 2 diabetes medications. The first drug in this class was Invokana (canagliflozin), first approved in 2013. After Invokana, a number of other SGLT2 inhibitors hit the market. Studies show they are effective in controlling excess blood glucose and lowering $\mathrm{A} 1 \mathrm{C}$ in people with Type 2 diabetes.

The drugs are rapidly growing in popularity. In the period between October 2014 and September 2015, doctors wrote 1.5 million prescriptions for Invokana or Farxiga (dapagliflozin), according to the FDA. They also come in extended formulas and in formulas combined with metformin.

These drugs work by preventing the kidneys from reabsorbing sugar and releasing it into the blood. But, what makes these medications so effective, could also contribute to a number of side effects. Beginning in December 2015, the FDA released a number of warnings linking these drugs to a number of side effects, some can be fatal. These range from common ones like yeast infections to more dangerous, rare problems like a link to increased bladder cancer risk and diabetic ketoacidosis-too much acid in the blood. ${ }^{1,2}$

Invokana is the most popular medication in this class, but there are four other FDA-approved SGLT2 inhibitors on the market. As FDA warnings continue to stack up against these drugs, SGLT2 lawyers are filing lawsuits on behalf of patients who say they suffered severe side effects from these drugs. Plaintiff say drug companies failed to warn them about the risks. ${ }^{3}$ releasing it into the blood. But, what makes these medications so effective, could also contribute to a number of side effects. Beginning in December 2015, the FDA released a number of warnings linking these drugs to a number of side effects, some can be fatal. These range from common ones like yeast infections to more dangerous, rare problems like a link to increased bladder cancer risk and diabetic ketoacidosis

\section{The mechanisms}

Sodium-glucose co-transporter-2 inhibitors work by inhibiting SGLT2 in the PCT, to prevent reabsorption of glucose and facilitate its excretion in urine. As glucose is excreted, its plasma levels fall leading to an improvement in all glycemic parameters. $^{4-6}$

This mechanism of action is dependent on blood glucose levels and, unlike the actions of thiazolidinediones (mediated through GLUTs), is independent of the actions of insulin. Thus, there is minimal potential for hypoglycemia, and no risk of overstimulation or fatigue of the beta cells. ${ }^{\text {? }}$ Because their mode of action relies upon normal renal glomerular-tubular function, SGLT2i efficacy is reduced in persons with renal impairment.

\section{Side effects}

Nvokana's side effects range from yeast infections to more serious issues like diabetic ketoacidosis. In May 2015 the FDA issued a drug safety communication that warned patients and doctors that studies linked Invokana and Invokamet — and other SGLT2 inhibitors - to diabetic ketoacidosis (DKA).

Unlike other SGLT2's, the FDA also found a link between Invokana and increased toe and foot amputations. The agency released a Drug Safety Communication in May 2016 and said it was investigating. Additionally, Invokana was not tested in pregnant women, but animal studies showed irreversible kidney problems in fetuses.

Despite the effectiveness of Invokana, it also has a notable list of side effects - some of which led plaintiffs to file lawsuits. 
Table 1 Side effects of SGLT2 inhibitiors ${ }^{8}$

\begin{tabular}{lcc}
\hline Urinary tract infections & Increased urination & Thirst \\
\hline Dry mouth & Male and female yeast infections & Constipation \\
Nausea & Abdominal pain & Fatigue \\
Loss of strength or energy & Hypersensitivity (rash, swelling) & Hypoglycemia \\
$\begin{array}{l}\text { Diabetic ketoacidosis } \\
\text { (DKA) }\end{array}$ & & \\
\end{tabular}

According to Janssen's news release, “Invokana is generally well tolerated, and the most common adverse events include genital yeast infection, urinary tract infections and changes in urination. The most common adverse reactions due to initiation of metformin, as noted in the prescribing information for that medication, are diarrhea, nausea, vomiting, flatulence, asthenia, indigestion, abdominal discomfort, and headache."

But, the FDA released several safety communications warning the public about a number of serious adverse event reports. The first came in May 2015, just two years after the drug's approval. The FDA warned Invokana and other SGLT2 inhibitors could increase the risk of diabetic ketoacidosis (DKA) a condition where too many toxins called ketones develop in the blood. DKA is a serious condition that requires hospitalization and may lead to coma or death.

Later that year, the agency released two more warnings linked to Invokana for increased bone-fracture risk and serious urinary tract infections. These infections could lead to blood and kidney infections, the FDA warned. Then, in 2016, the agency strengthened the kidney damage warning for Invokana and another SGLT2 drug, Farxiga ${ }^{8}$ (table 1).

In 2016, the FDA also began investigating reports of acute pancreatitis linked to SGLT2 inhibitors. Acute pancreatitis occurs when the pancreas, the organ responsible for producing insulin, becomes swollen and inflamed. It can cause serious health issues if not treated and may even be fatal. Currently, the agency is "evaluating the need for regulatory action."

Invokana is available in $100 \mathrm{mg}$ and $300 \mathrm{mg}$ tablets. People with moderate renal problems should not take more than $100 \mathrm{mg}$ daily, and doctors do not recommend Invokana for people with poor kidney function. Some side effects were more severe with higher doses.

\section{CONCLUDING REMARKS}

Though SGLT2 inhibitors represent a novel class of drugs which will certainly help a large number of people with diabetes achieve target control in a safe and well-tolerated manner, they pose serious life threatening side effects in certain population of diabetics. Only long term studies and further post-marketing surveillance will decide the fact that these class of medications will be a sustainable antidiabetics like metformin or sulfonylureas. Their unique mechanism of action, coupled with pleiotropic benefits on weight and blood pressure, should make them attractive choices for add-on therapy to persons not controlled on other medications.

\section{REFERENCES}

1. Abdul GM, DeFronzo R. Dapagliflozin for the treatment of type 2 diabetes. Expert Opin Pharmacother. 2013;14:16951703. doi: 10.1517/14656566.2013.812632. [PubMed] [Cro ss Ref]

2. Bailey CJ. Renal glucose reabsorption inhibitors to treat diabetes. Trends Pharmacol Sci. 2011;32:63-71. doi: 10.1016/j.tips.2010.11.011

3. Kasichayanula S, Liu X, LaCreta F, Griffen S, Boulton D. Clinical pharmacokinetics and pharmacodynamics of dapagliflozin, a selective inhibitor of sodium-glucose co-transporter type 2. Clin Pharmacokinet. 2014;53:1727. doi: $10.1007 / \mathrm{s} 40262-013-0104-3$

4. Wright EM. Renal Na+-glucose cotransporters. Am J Physiol Renal Physiol. 2001;280(1):F10-F18. [PubMed]

5. Lee YJ, Lee YJ, Han HJ. Regulatory mechanisms of $\mathrm{Na}(+) /$ glucose cotransporters in renal proximal tubule cells. Kidney Int Suppl. 2007;(106):S27-35. [PubMed]

6. Hummel CS, Lu C, Loo DD, Hirayama BA, Voss AA, Wright EM. Glucose transport by human renal NA+/ d-glucose cotransporters SGLT1 and SGLT2. Am J Physiol Cell Physiol. 2011;300:C721. doi: 10.1152/ajpcell.00388.2010. [PMC free article] [PubMed] [Cross Ref]

7. Nauck MA. Update on developments with SGLT2 inhibitors in the management of type 2 diabetes. Drug Des Devel Ther. 2014;8:1335-1380. doi: 10.2147/DDDT.S50773

8. SGLT2 Inhibitors https://www.drugwatch.com/sglt2-inhibitors(Accessed on $15^{\text {th }}$ July 2016)

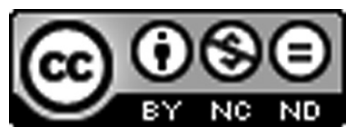

This work is licensed under a Creative Commons Attribution-Non Commercial-No Derivatives 4.0 International License. To view a copy of this license, visit http://creativecommons.org/licenses/by-nc-nd/4.0/ 